\title{
Research progress of low resolution radar target recognition technology
}

\author{
Zhi-qiang Li,Zi-zhi Gaoand Jun-tao Ma \\ Ordnance Engineering College \\ 121500045@qq.com
}

Keywords:low resolution radar;recognition technology;research status

\begin{abstract}
Radar is an electronic device for radio detection and ranging. High resolution radar ofultra wideband will be the developing trend of new generation radar.But because of the long project time, high manufacture cost and key technologies unsolved. Thus it cannot replace the low resolution radar in the short term.And this paper introduces the research status of low resolution radar target recognition.
\end{abstract}

\section{Introduction}

Radar is an electronic device for radio detection and ranging. Conventional radar irradiate target by radiating electromagnetic wave and obtain the target information such asdistance, distance change rate (radial velocity), height, direction and so on according to the received radar echo. With the rapid development of radar technology, radar function has been developed from the basic function of target detection, target location and target tracking. In short, the radar target recognition is an inverse process of electromagnetic scattering, that is to identify target though backward electromagnetic scattering.The radar target recognition system generally pre processing and extract feature from the echo signal received, and then compared with the existing feature template library based on the prior data, so as to get the target's category, size and other related information.

\section{Low resolution radar target recognition technology}

\subsection{Research significance of Low resolution radar target recognition}

High resolution radar ofultra wideband will be the developing trend of new generation radar.But because of the long project time, high manufacture cost and key technologies unsolved. Thus it cannot replace the low resolution radar in the short term. Low resolution radar has many advantages itself. The mature technology, lowprice relatively, and some work in the Mi Bo radar has a good anti stealth ability and anti radiation missile capability.As a result, the low resolution radar plays anirreplaceable role in many fields compared with high resolution radar. Meanwhile, generalactive radar in our country is low resolution radar without radial and lateral high resolution. Although the target information received from low resolution radar echo is very limited, cannot achieve the goal of precise identification, some intrinsic characteristics of target can affect the target echo.According to a large number of domestic and foreign studies, it is proved that the rough classification of target is feasible in the condition of low resolution. Therefore, there is great practical significance to study the target recognition of low resolution radar.

\section{2 research status of low resolution radar target recognition}

\subsubsection{Foreign research status of low resolution radar target recognition}

(1) The US Air Force Research Laboratory, Air Force Institute, Air Force Academy dedicated to the study of aircraft target identification, from the aircraft's RCS feature extraction to the establishment of statistical invariant features, and has been improving the target resolution and identification radar. The United States Navy research laboratory has increased the correlation processing equipment of the wideband linear frequency modulation signal, and got a higher resolution target range image, obtained the good recognition effect. [1]

(2) The Americanprofessor has designed a conventional low resolution radar target recognition 
system based on multi feature decision space method, and realized the real-time identification of a certain aircraft by the combination of speed, size and magnitude. In 1995s, Canadian doctor Leung designed an intelligent recognition system of sea level warning radar for the defense research institution in Ottawa.[2] Then Dr Leung constructed the target tracking classifier for low resolution air defense radar and extracted featuressuch as target attribute, height, speed and acceleration, realizing theclassification of five types of target airplane. [3]

(3) Using target echo intensity of low resolution radar and its fluctuation characteristics and modulation spectrum characteristics to identify helicopter. Georgia Institute in American missile command studiedthe Doppler frequency shift characteristics of the rotor plane, and analyzes the echo of 8 kinds of helicopters. Warsaw university in Poland analyzes the echo of four kinds of helicopter with S- band pulse compression.[4] The study shows that the periodic modulation spectrum of different targets is very distinct, so it can be used for target recognition. 。

\subsubsection{Domestic research status of low resolution radar target recognition}

Compared with foreign countries, the research on the low resolution radar targetrecognition has a late start. Some units such as National University of Defense Technology, Harbin Institute of Technology, Xi'an Electronic and Science University have carried out some theoretical research for the low resolution radar target recognition, and give a number of feasible target recognition methods. According to the characteristics of fine structure of radar target echo amplitude, Guo Guirong[5]etc of National Defense Science and Technology University propose using Fourier transform and Mellin transform binary coding sequence and extracts the symbol characteristic of echo signal, combined with fuzzy pattern recognition technology to complete the recognition of maritime targets.

Ji Zhenyuan[6] etc of Harbin Industrial University analyze the target feature of battlefield reconnaissance radar. They propose usingthe target's instantaneous frequency variation characteristics to achieve the distinction between the maneuvering and non maneuvering targets.

Wang Wei etc[7]of National University of Defense Technology research on the low resolution radar target echo. The waveform of target echo brings closely related information with the target characteristics. They propose using three kinds of characteristics, such as the quasi steady state information, significant state feature information,the changing relationship feature information, to reflecttarget waveform. Xin Yulin etc[8] of National University of Defense Technology analyze the waveform characteristics of the aircraft target echo. They extract the entropy value based on the waveform and the characteristic quantities of grayscale, combined with neural network, realize the classification and identification of aircraft targets and prove the effectiveness of feature extraction and classification recognition using target echo waveform.

Radar echo is a typical non-stationary signal. So some research unitsapplied the feature extraction algorithm based on wavelet decomposition to the low resolution radar target recognition system. They take advantages of wavelet transform to decompose the target echo signal, extract the wavelet coefficients of different scales and combine with neural network for recognition. All shows they achieved good results.

\section{Summary}

In summary, the research of low resolution radar target recognition can be summarized as follows: First, low resolution radar target recognition mainly reveals the electromagnetic scattering characteristics of the target. Second, the scattering information acquisition of Lowresolution radar target is mainly dependent on the continuous rise of hardware level, it can be used to process more information in the same time. There is still a lot of developing space in low resolution target recognition in the future. With more excellent hardware, mature technology, whether from the method side or hardware side, there will be more ways to identify the target. 


\section{Reference}

[1]G Linde.Use of Wide-band Waveforms for Target Recognition with Surveillance Radar[C].Proceedings of IEEE International radar conference, 2000.

[2]H Leungetal.Intelligent Radar Recognition System for Surveillance[C].Proceedings of IEEE International Conference on Systems, Manand Cybernetics1995: 2280 2285.

[3]H Leungetal.Bayes ianand Dempster-Shafer Target Identification for Radar Surveillance[J]. IEEE Trans on AES.2000, 36 (2): 432-447.

[4]JMisiurewiczetal.Analysis of Recorded HelicopterEcho[J].Proceedings of IEEE Radar-97: 449 451.

[5]Guo gui-rong, yu wen-xian, hu bu-fa.A kind of effective new technology of ship target recognition[J].Systems engineering and electronics, 1996 The sixth period: 1-7.

[6]Ji zhen-yuan, Meng xian-de.Automatic identification of battlefield reconnaissance radar target[J].Journal of Harbin institute of technology university, 2001，33 (6): 830-833.

[7]Wang wei, Zhang han-hua, Jiang wei-dong etc.Low resolution radar target feature extraction method[J].Journal of national university of defense technology, 2002, 24 (2): 31-35.

[8]Xin yu-lin , $\mathrm{Xu}$ ren-can.The low resolution radar target recognition methed research[J].Communications and information technology, 200519 of the period: 17-20.

[9]Zhang jing-yuan, Zhang bing, Jiang xing-zhou.Feature extraction method based on wavelet transform analysis[J].The signal processing, 2000, 16 (2): 156-162. 